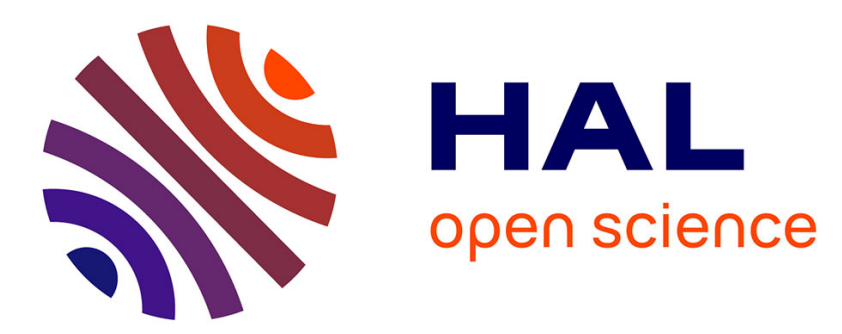

\title{
Numerical Study of the Current Constriction in a Vacuum Arc at Large Contact Gap
}

Benoît Tezenas Du Montcel, P Chapelle, Christophe Creusot, Alain Jardy

\section{To cite this version:}

Benoît Tezenas Du Montcel, P Chapelle, Christophe Creusot, Alain Jardy. Numerical Study of the Current Constriction in a Vacuum Arc at Large Contact Gap. IEEE Transactions on Plasma Science, 2019, 47 (5), pp.2765-2774. 10.1109/TPS.2019.2909964 . hal-02130285

\section{HAL Id: hal-02130285 \\ https://hal.science/hal-02130285}

Submitted on 13 Jan 2022

HAL is a multi-disciplinary open access archive for the deposit and dissemination of scientific research documents, whether they are published or not. The documents may come from teaching and research institutions in France or abroad, or from public or private research centers.
L'archive ouverte pluridisciplinaire HAL, est destinée au dépôt et à la diffusion de documents scientifiques de niveau recherche, publiés ou non, émanant des établissements d'enseignement et de recherche français ou étrangers, des laboratoires publics ou privés. 
123 . 


\title{
Numerical Study of the Current Constriction in a Vacuum Arc at Large Contact Gap
}

\author{
Benoît Tezenas du Montcel, Pierre Chapelle, Christophe Creusot, and Alain Jardy
}

\begin{abstract}
A classical two temperature magnetohydrodynamic modelling approach is used to study the influence of a large contact gap (up to $40 \mathrm{~mm}$ ) on the behaviour of a diffuse vacuum arc controlled by a $5 \mathrm{mT} / \mathrm{kA}$ uniform external axial magnetic field between two copper electrodes with a $20 \mathrm{~mm}$ radius. The current constriction and energy flux density at the anode surface are more particularly analyzed, considering both supersonic and subsonic flow conditions. In the case of a supersonic arc, simulations show that the constriction of the current develops in the whole interelectrode region, but the constriction level at the anode surface does not evolve monotonically with the contact gap. The constriction is partly correlated to the radial compression of the plasma. In the case of a subsonic arc, the current constriction is related to the presence of the anode sheath. It occurs only close to the anode (from a constant distance from the anode of around $15 \mathrm{~mm}$ ). Whereas the current constriction at the anode surface increases when the gap length goes from $10 \mathrm{~mm}$ to $20 \mathrm{~mm}$, it no longer evolves when increasing the gap length from $20 \mathrm{~mm}$ to $40 \mathrm{~mm}$. For both flow conditions, the evolution with the gap length of the radial profile of the energy flux density transferred by the plasma to the anode is similar as that followed by the current constriction at the anode.
\end{abstract}

Index Terms-Numerical simulation, diffuse vacuum arc, axial magnetic field, large contact gap.

\section{Introduction}

High-current vacuum arc (VA) in diffuse mode has been the subject of a great number of research studies through the years for its applications in circuit-breakers (CB) [1]. Whereas the diffuse mode is characteristic of a free burning VA at low currents (up to a few kA), at higher currents an anode spot appears and VA constricts taking the form of a columnar arc, which strongly decreases the breaking capability of the circuit-breaker. Applying an axial magnetic field (AMF) may be used to distribute more evenly the arc energy on the anode surface, which allows to significantly increase the current at which the columnar arc mode develops [2]. Nowadays, vacuum circuit-breakers based on the application of an AMF have become a key technology for Medium-Voltage applications [3]. For HighVoltage applications, circuit-breakers rely mostly on the use of SF6 as an interrupting medium. This gas offers among others excellent dielectric performances. However, it has major harmful impacts to the environment. This

B. Tezenas du Montcel was with the Institut Jean Lamour, UMR 7198 CNRS/Universite de Lorraine, Laboratory of Excellence DAMAS, 2 allee Andre Guinier, BP 50840, 54011 Nancy Cedex, France and also with the SuperGrid Institute, 23 rue Cyprian, BP 1321, 69611 Villeurbanne, France.

P. Chapelle and A. Jardy are with the Institut Jean Lamour.

C. Creusot is with the SuperGrid Institute. induces an important pressure to substitute SF6 by a more environmentally friendly breaking medium. Among the technologies that are considered, vacuum is a valuable candidate [4].

Knowledge on high-current diffuse vacuum arc has made great progress for the last fifteen years thanks to a significant number of numerical studies. Most of these studies are based on relatively similar magnetohydrodynamic (MHD) descriptions of the diffuse plasma column using a two temperature representation of the plasma [2], [5]-[12], [14], [15]. A large number of these works were inspired by the model presented by Schade and Shmelev in 2003 [2]. These authors developped a 2D axisymmetric model considering energy transfers (including radiation energy) and the actions of the self-magnetic field and an externally imposed AMF. Both supersonic (at low arc current) and subsonic (at high arc current) plasma flow conditions were accounted for. In [5], the emission of metal vapor at the anode surface and its interaction with the plasma created at the cathode were included, revealing that the metal vapor was fully ionized very close to the anode. Hartmann et al. [8] were the first to attempt the modelling of the diffuse plasma column based on a 3D geometry, yet the anode was treated as a passive collector of particles. Other significant works include the series of models reported by Wang et al. and applied first to the study of the influence of various AMF radial profiles on the behaviour of a supersonic arc [6]. Later, their model was extended to account for a $3 \mathrm{D}$ geometry [11] and the effects of a realistic AMF profile was in particular investigated [13]. Recently, their work was combined with a thermal model of the anode to consider particles emitted at the anode [14]. One should mention also the work of Langlois et al. [10] having strong similarities with the model reported in [6] and which was used to investigate in detail the various components of the energy flux density transferred to the anode for both supersonic and subsonic flow conditions. Finally, using a significantly different approach from all above works, Londer and Ulyanov [7] have developed a semianalytical model of the VA.

Until recently, most models were applied to simulate the case of a short contact gap of $10 \mathrm{~mm}$ or less, representative of the conditions in a Medium-Voltage CB [16]. For HighVoltage levels, the contact gap can rise up to $50 \mathrm{~mm}$ [16]. The behavior of VA in this range of contact gap is still not well understood and the numerical studies of VA in High-Voltage CB stay rare. To the best of our knowledge, only two of such studies are available in the literature. In 
[12], the constriction of VA in a large contact gap $(40 \mathrm{~mm})$ under various AMF radial profiles was simulated using a 3D model. More recently, Han et al. [15] have performed, on the basis of a 2D model, a detailed study of the influence of a large contact gap (which was varied in the $10-50 \mathrm{~mm}$ range) on the behavior of the arc column. In this work, the radial and axial components of the self-magnetic field were neglected and only the case of a subsonic VA was treated.

In this paper, a classical 2D and two temperature MHD model, in which the three components of the selfmagnetic field are accounted for, is used to analyze the effects produced by a large contact gap $(10-40 \mathrm{~mm})$ on the constriction of a high-current diffuse VA between copper contacts, considering both supersonic and subsonic plasma flow conditions. We first describe the numerical model, including the model assumptions and the definition of the boundary conditions. Next, simulation results focusing in particular on the evolution of the current density in the interelectrode region are given under supersonic and subsonic conditions and the different phenomena governing the current constriction under each condition are discussed.

\section{Magnetohydrodynamic Model}

A diffuse $\mathrm{VA}$ is a complex multi-scale phenomenon, which can be divided into different regions (Fig. 1) [2]. The plasma is emitted at the cathode surface by small size and very mobile cathode spots. The plasma jets produced by the spots mix near the cathode to form a continuous plasma column. The next region is the hydrodynamic region, inside which the plasma flows in direction to the anode. Finally, before being collected by the anode, the plasma crosses a near anode space charge sheath. In this paper, only the hydrodynamic region is treated with a MHD approach. Near cathode and near anode regions are considered as very thin compared to the hydrodynamic region and the phenomena taking place in these regions are accounted for through the boundary conditions of the model.

Based on the experimental observations regarding VAs controlled by a strong AMF at large contact gaps reported in [17], the model developed in this paper uses as a first approximation a 2D-cylindrical geometry. As shown in Fig. 2, the computational domain consists of three subdomains: the electrodes (cathode and anode), the interelectrode region enclosing the plasma and a lateral vacuum region separated from the interelectrode region by a impermeable fictitious boundary. The consideration of a lateral vacuum region aims at moving away the external boundaries of the domain in order to facilitate the definition of the boundary conditions on these boundaries. The electromagnetic variables are computed in the three regions, whereas the hydrodynamic variables are computed only in the interelectrode region. The model was implemented in the numerical simulation software Comsol-Multiphysics@.

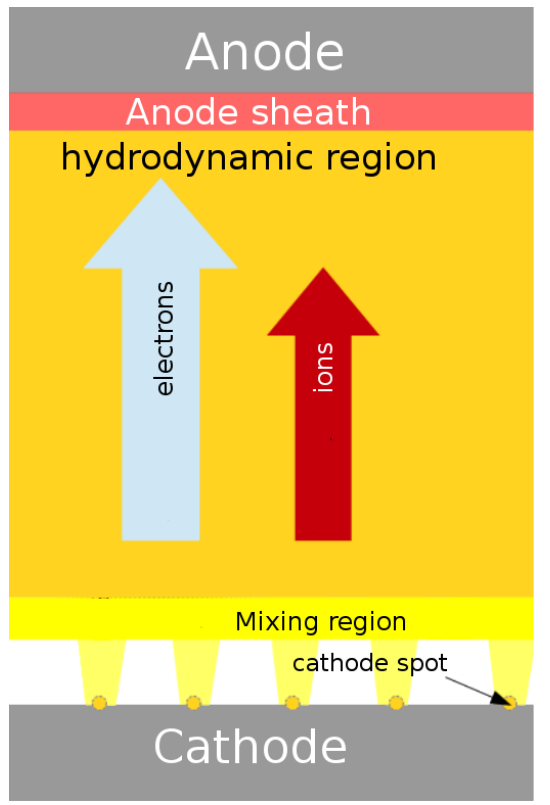

Fig. 1: Sketch of the diffuse VA (not to scale).

External anode

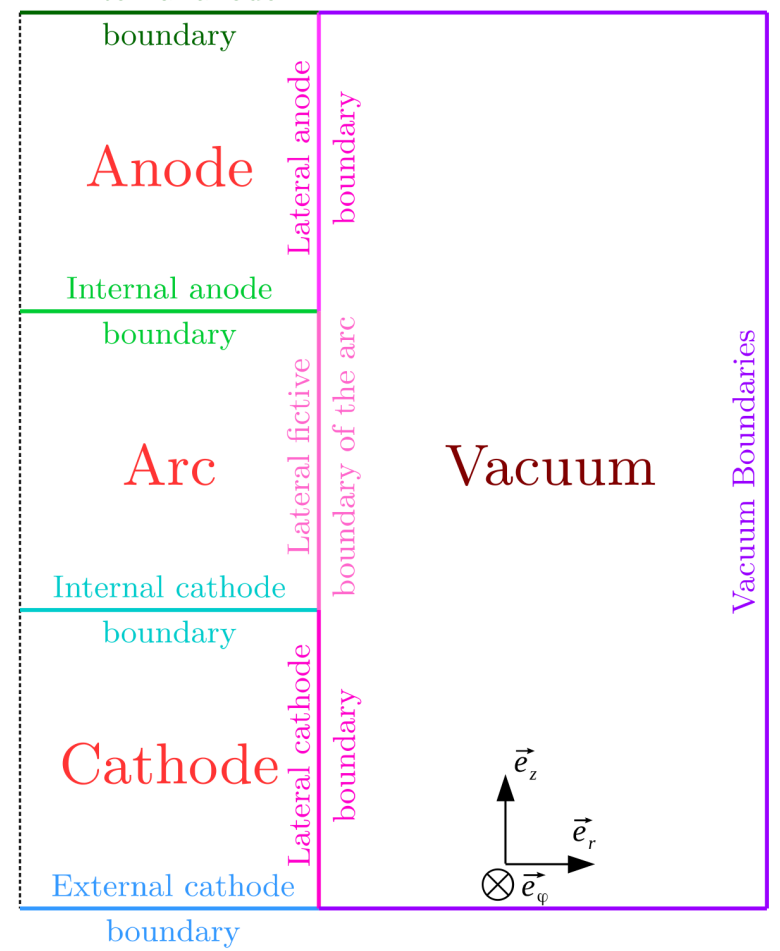

Fig. 2: Computational domain.

\section{A. Physical model}

The plasma is represented as a mixture of two fluids, namely copper ions and electrons with respective masses $m_{i}$ and $m_{e}$, whose behavior is modeled using the conservation equations established by Braginskii [18] coupled with the Maxwell equations. This group of equations can be simplified with the aid of various classical approximations:

- For an Alternative-Current with a $50 \mathrm{~Hz}$ frequency, 
the time scale of the variation of the current is very large compared to the time scales associated to the plasma flow. Consequently, the plasma may be described using a steady state approach.

- Because the Debye length is very small in front of the typical length scale of the system [10], the plasma may be considered as quasi-neutral.

- Ionization and recombination collisions in the hydrodynamic region are negligible [2]. Hence the average ion charge is considered as a fixed value $(Z=1.85$ for copper electrodes [19]).

- Electron inertia is neglected.

- Both ions and electrons are assumed to be unmagnetized particles and are treated as ideal gases.

- In a diffuse VA, the radiative dissipation can be neglected [2].

- The emission of vapor particles at the anode is not considered. Indeed, the simulation results presented in [2] have shown that the anode starts to become active only for very high current density $\left(>1300 \mathrm{~A} / \mathrm{cm}^{2}\right.$, which corresponds to a current intensity of about $16 \mathrm{kA}$ for $20 \mathrm{~mm}$ radius electrodes). Note however that for some anode materials with a low melting point and high vapor pressure (e.g. brass) the anode emits vapor particles even at low current densities [20].

Based on these approximations and some manipulations of the Braginskii and Maxwell equations, a new set of equations may be established. This new set of equations and the variables that may be deduced from each equation are detailed below.

- The ion density $\left(n_{i}\right)$, velocity $\left(\vec{u}_{i}\right)$ and temperature $\left(T_{i}\right)$ may be obtained from the equations of conservation of particles, momentum and energy relative to the ion flow.

$$
\left\{\begin{aligned}
& \vec{\nabla} \cdot n_{i} \vec{u}_{i}=0 \\
& m_{i} n_{i}\left(\vec{u}_{i} \cdot \vec{\nabla}\right) \vec{u}_{i}=-\vec{\nabla}\left(p_{i}+p_{e}\right)-\vec{\nabla} \cdot \overline{\bar{\pi}}_{i} \\
&+\vec{J} \times \vec{B} \\
& \frac{5}{2} n_{i} \vec{u}_{i} \cdot \vec{\nabla} k_{b} T_{i}-\vec{u}_{i} \cdot \vec{\nabla} p_{i}=\vec{\nabla} \cdot k_{i} \vec{\nabla} T_{i} \\
&-\overline{\bar{\pi}}_{i}: \vec{\nabla} \vec{u}_{i}+Q_{i}
\end{aligned}\right.
$$

- The density of electrons $\left(n_{e}\right)$ can be calculated from the relation of electroneutrality, their velocity $\left(\vec{u}_{e}\right)$ can be deduced from the current density definition and their temperature $\left(T_{e}\right)$ is given by the equation of conservation of the electron energy.

$$
\left\{\begin{array}{l}
n_{e}=Z n_{i} \\
\vec{u}_{e}=\vec{u}_{i}-\frac{\vec{J}}{e n_{e}} \\
\frac{5}{2} n_{e} \vec{u}_{e} \cdot \vec{\nabla} k_{b} T_{e}-\vec{u}_{e} \cdot \vec{\nabla} p_{e}=\vec{\nabla} \cdot k_{e} \vec{\nabla} T_{e} \\
+\frac{\vec{J}^{2}}{\sigma}-Q_{i}
\end{array}\right.
$$

- The current density $\vec{J}$ can be calculated from the generalized Ohm law, which is deduced by reformulating the equation of the conservation of the electron momentum.

$$
\vec{J}=\sigma\left[-\vec{\nabla} V+\vec{u}_{e} \times \vec{B}+\frac{\vec{\nabla} p_{e}}{e n_{e}}+0.9 \frac{k_{b}}{e} \vec{\nabla} T_{e}\right]
$$

- The electric and magnetic fields $(\vec{E}, \vec{B})$ are calculated from the electric and magnetic vector potentials $(V, \vec{A})$. The latters can be obtained in the plasma from the following equations derived from the combination of Eq. (3) with respectively the equation of conservation of the current and the equation of Maxwell-Ampere:

$$
\left\{\begin{array}{c}
\vec{\nabla} \cdot \sigma \vec{\nabla} V=\vec{\nabla} \cdot \sigma\left(\frac{\vec{\nabla} p_{e}}{e n_{e}}+0.9 \frac{k_{b}}{e} \vec{\nabla} T_{e}\right) \\
+\vec{\nabla} \cdot \sigma\left(\vec{u}_{e} \times \vec{B}\right)
\end{array}\right.
$$

- In the electrode and vacuum regions, $V$ and $\vec{A}$ are derived from the following equations:

$$
\left\{\begin{array}{l}
\vec{\nabla} \cdot \sigma \vec{\nabla} V=0 \\
\frac{1}{\mu_{0} \sigma} \vec{\Delta} \vec{A}=-\vec{\nabla} V
\end{array}\right.
$$

In this set of equations, $p_{i}$ and $p_{e}$ are the ionic and electronic pressures, respectively; $\overline{\bar{\pi}}_{i}$ is the ion viscous stress tensor; $k_{i}$ and $k_{e}$ are the ionic and electronic thermal conductivities, respectively; $Q_{i}$ is the energy received by the ions during collisions with electrons and $\sigma$ is the electrical conductivity. $k_{b}$ is the Boltzmann constant, $e$ is the elementary positive charge and $\mu_{0}$ is the magnetic permeability of the vacuum. $\overline{\bar{\pi}}_{i}, k_{i}, k_{e}, Q_{i}$ and $\sigma$ in the plasma region are respectively given by:

$$
\begin{gathered}
\overline{\bar{\pi}}_{i}=0.96 n_{i} k_{b} T_{i} \tau_{i}\left[\left(\vec{\nabla} \vec{u}_{i}+\vec{\nabla} \vec{u}_{i}^{\dagger}\right)-\frac{2}{3} \vec{\nabla} \cdot \vec{u}_{i} \overline{\bar{I}}_{d}\right] \\
Q_{i}=3 \frac{m_{e}}{m_{i}} \frac{n_{e}}{\tau_{e}} k_{b}\left(T_{e}-T_{i}\right) \\
\left\{\begin{array}{l}
k_{i}=3.9 \frac{n_{e} k_{b}^{2} T_{i} \tau_{i}}{m_{i}} \\
k_{e}=4.9 \frac{n_{e} k_{b}^{2} T_{e} \tau_{e}}{m_{e}} \\
\sigma=2.27 e^{2} \frac{n_{e} \tau_{e}}{m_{e}}
\end{array}\right.
\end{gathered}
$$

$\overline{\bar{I}}_{d}$ is the identity tensor and $\tau_{e}$ and $\tau_{i}$ are the collisional relaxation times of electrons and ions:

$$
\left\{\begin{array}{c}
\tau_{e}=\frac{12 \pi^{3 / 2}}{\sqrt{2}} \frac{\varepsilon_{0}^{2} \sqrt{m_{e}\left(k_{b} T_{e}\right)^{2}}}{n_{e} e^{4} Z \ln \Lambda} \\
\tau_{i}=\frac{12 \pi^{3 / 2}}{\sqrt{2}} \frac{\varepsilon_{0}^{2} \sqrt{m_{i}\left(k_{b} T_{i}\right)^{2}}}{n_{e} e^{4} Z^{4} \ln \Lambda}
\end{array}\right.
$$

with $\varepsilon_{0}$ the electric permittivity and $\ln \Lambda$ the Coulomb logarithm. Numerical values in Eq. (3), (4), (6), (8) and (9) are parameters which appear in the expressions of the transport coefficients derived by Braginskii [18]. They are dependent on the ion charge. The values used here correspond to the case $\mathrm{Z}=2$. 


\section{B. Boundary conditions}

a) Electromagnetic variables: On all external boundaries of the computational domain, the magnetic vector potential is fixed as:

$$
\vec{A} \times \vec{n}=\overrightarrow{0}
$$

with $\vec{n}$ the normal vector to the external boundary.

On the external boundary of the cathode, the normal current density, which is assumed uniform, is specified by the condition:

$$
\vec{\nabla} V \cdot \vec{n}=-\frac{I_{\text {arc }}}{\pi R^{2}}
$$

where $I_{\text {arc }}$ is the arc current and $R$ is the radius of the contacts. The voltage on the external boundary of the anode is fixed to ground $(0 \mathrm{~V})$. On the external boundaries of the vacuum region, the condition that no current flows through these boundaries takes the form:

$$
\vec{\nabla} V \cdot \vec{n}=0
$$

On the boundary between the cathode and the VA, the profile of the normal current density $J_{c}$ is imposed to take into account the cathode spot distribution upon the cathode surface. In this paper, the profile of the normal current density on the cathode surface is considered uniform, like on the cathode external boundary (Eq. (12)).

On the boundary between the anode and the VA, the normal current density $J_{a}$ is imposed to take into account the influence of the anode sheath [21]:

$$
J_{a}=J_{t h} e^{-\frac{e \varphi_{s h}}{k_{b} T_{e}}}+Z e n_{i} u_{i z}
$$

with $J_{t h}$ the electron thermal current given by:

$$
J_{t h}=-\frac{1}{4} e n_{e} \sqrt{\frac{8}{\pi} \frac{k_{b} T_{e}}{m_{e}}}
$$

and $\varphi_{s h}$ the voltage drop in the anode sheath. Considering that the anode surface is equipotential $\left(V=V_{0}\right)$, the anode sheath voltage drop is related to $V_{0}$ and to the electric potential at the boundary between the hydrodynamic region and the anode sheath $V_{a}$ through the relation $\varphi_{s h}=V_{0}-V_{a}$. The potential $V_{0}$ is a constant that may be computed by equating the total electron current emitted at the cathode to the total electron current collected at the anode. The boundaries between the vacuum region and respectively the plasma and the electrodes are treated as impermeable to the electric current.

b) Hydrodynamic variables: Boundary conditions for supersonic (at low current) and subsonic (at high current) flow conditions have to be treated separately. Transition between supersonic and subsonic conditions happens for a Mach number $M a=1$, with $M a$ given by:

$$
M a=\frac{u_{i z}}{\sqrt{\frac{k_{b}}{m_{i}}\left(\gamma T_{i}+T_{e}\right)}}
$$

where $\gamma$ is the adiabatic index. From the data gathered by Schade and Shmelev [2], the critical current density separating supersonic and subsonic flow conditions is about $300 \mathrm{~A} / \mathrm{cm}^{2}$, which corresponds to a current intensity of about $3800 \mathrm{~A}$ for $20 \mathrm{~mm}$ radius electrodes.

The conditions specified on the boundary between the cathode and the plasma are as follows. For supersonic conditions, three variables relative to the ion flow are imposed, namely the ion temperature, the ion velocity and the ion pressure, which may be expressed as a function of the ion erosion rate of the cathode $\mu_{e r}$ as follows:

$$
p_{i}=-\frac{\mu_{e r} J_{c}}{m_{i} u_{i z}} k_{b} T_{i}
$$

For subsonic conditions, only two variables relative to the ion flow need to be imposed. The variables retained in the present model are the ion temperature and the ion mass flow rate, which is given by:

$$
m_{i} n_{i} u_{i z}=-\mu_{e r} J_{c}
$$

Regarding the flow of electrons, it is necessary to specify the electron temperature.

Conditions specified on the boundary between the anode and the plasma are as follows. Boundary conditions relative to the ion flow are required only in the case of subsonic conditions. Our model uses a condition on the ion pressure. This condition is derived from global mass and energy balances across an intermediate region, a presheath, between the hydrodynamic region and the anode sheath, assuming that the Bohm criterion [22] is marginally fullfilled at the entrance of the anode sheath.

Regarding the flow of electrons, a Fourier condition for the electron energy conservation equation is obtained by considering a global energy balance through the near anode region (presheath and sheath) [2]. This condition takes the form:

$$
k_{e} \frac{\partial T_{e}}{\partial z}=n_{e} u_{e z}\left(\frac{k_{b} T_{e}}{2}+e \varphi_{s h}\right)-0.9 \frac{k_{b} T_{e}}{e} J_{a}
$$

Finally, the lateral boundary of the plasma region is treated as an adiabatic and stress free wall.

\section{Supersonic arc}

In this section, the behavior of a supersonic diffuse arc between two contacts of $20 \mathrm{~mm}$ radius with a current $I_{\text {arc }}=1 \mathrm{kA}$ and controlled by a uniform AMF of strength $B_{A M F}=5 \mathrm{mT}$ is simulated for a range of gap lengths varying from $10 \mathrm{~mm}$ to $40 \mathrm{~mm}$. The simulations have been performed setting at the boundary between the cathode and the plasma the ion temperature to $0.2 \mathrm{eV}$ [24], the ion velocity (assumed to be purely axial) to $10^{4} \mathrm{~m} / \mathrm{s}$ [19], the ion erosion rate to $50 \mu \mathrm{g} . \mathrm{C}^{-1}[23]$ and the electron temperature to $1.5 \mathrm{eV}[25]$.

\section{A. Results}

The norm and the streamlines of the projection of the current density vector onto the $(\mathrm{r}, \mathrm{z})$ plane for $10 \mathrm{~mm}$ and $40 \mathrm{~mm}$ gaps are shown in Fig. 3. For $10 \mathrm{~mm}$, the current experiences an important constriction in front of the cathode, which gets stronger as the plasma flows from cathode to anode. In contrast, for $40 \mathrm{~mm}$, the 
current constriction remains weak until a distance of about $20 \mathrm{~mm}$ from the cathode. Beyond this distance, it strongly increases in direction to the anode. Note that for both gap lengths a reduction of the current constriction is observed very close (within $5 \mathrm{~mm}$ ) to the anode.

(a)
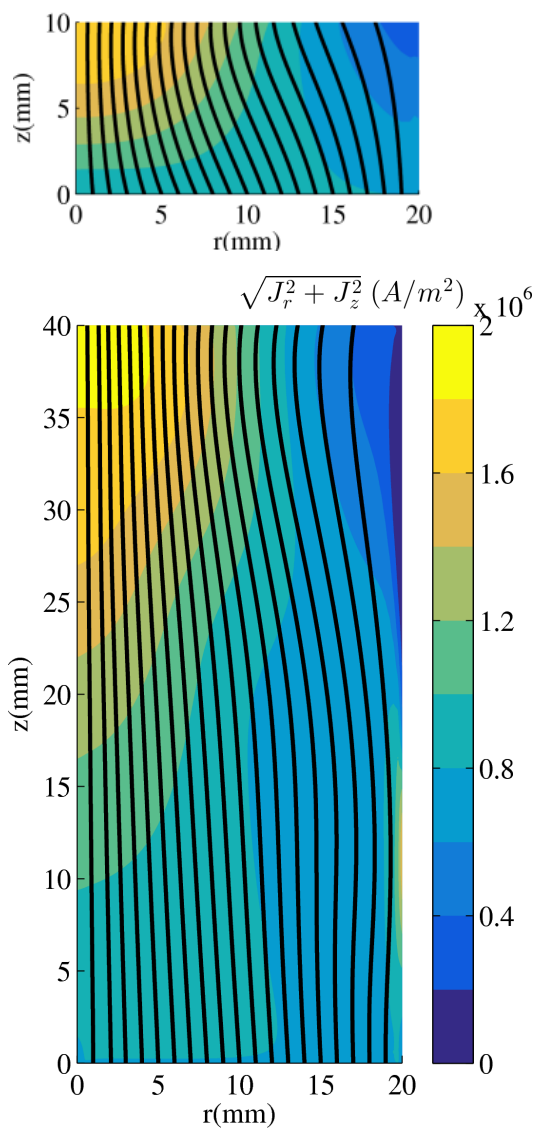

Fig. 3: Distribution of the norm and streamlines of the projection of the current density vector onto the $(\mathrm{r}, \mathrm{z})$ plane for (a) $L_{a r c}=10 \mathrm{~mm}$ and (b) $L_{a r c}=40 \mathrm{~mm}$ (supersonic arc, $I_{\text {arc }}=1 \mathrm{kA}$ and $\left.B_{A M F}=5 \mathrm{mT}\right)$. The coordinate $\mathrm{z}=0$ corresponds to the cathode surface. .

In the following, let us define the constriction rate $\tau_{c}$ as the fraction of the current passing through a disk with a $R / \sqrt{2}$ radius:

$$
\tau_{c}(z)=-\frac{2 \pi}{I_{a r c}} \int_{0}^{R / \sqrt{2}} r J_{z}(r, z) d r
$$

Fig. 4 shows the axial profile of $\tau_{c}$ for different gap lengths. At the cathode boundary, the value of $\tau_{c}$ is 0.5 , since the area of the disk with a $R / \sqrt{2}$ radius represents half of that of the contact surface and the distribution of the current density is considered to be uniform. For a $10 \mathrm{~mm}$ gap, $\tau_{c}$ increases almost linearly and reaches 0.73 at the anode boundary. With the increase of $L_{\text {arc }}$, three phases in the evolution of $\tau_{c}$ can be progressively differentiated. Close to the cathode, the growth of the constriction rate is weak. Next, the growth gets strong. Finally, in the near anode area the growth of $\tau_{c}$ becomes weak again. For gap lengths greater than $30 \mathrm{~mm}$, the constriction rate even begins to decrease in the immediate vicinity of the anode. For a $40 \mathrm{~mm}$ gap, $\tau_{c}$ reaches a maximum value of 0.77 and then decreases to 0.75 at the anode boundary.

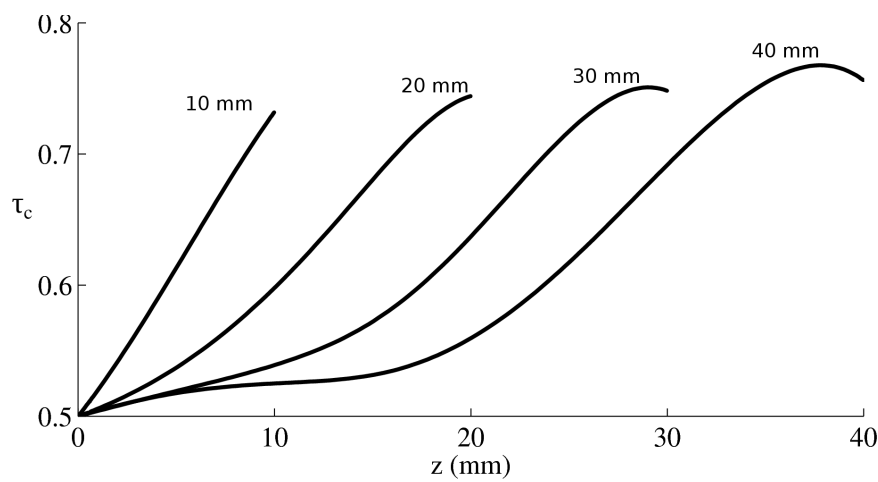

Fig. 4: Axial profile of the constriction rate $\tau_{c}$ for different gap lengths (supersonic arc, $I_{a r c}=1 \mathrm{kA}$ and $B_{A M F}=$ $5 \mathrm{mT}$ ).

The radial profile of the axial component of the current density on the anode surface for different gap lengths is shown on Fig. 5a. Current density is normalized by the cathode current density $J_{c}$.

(a)

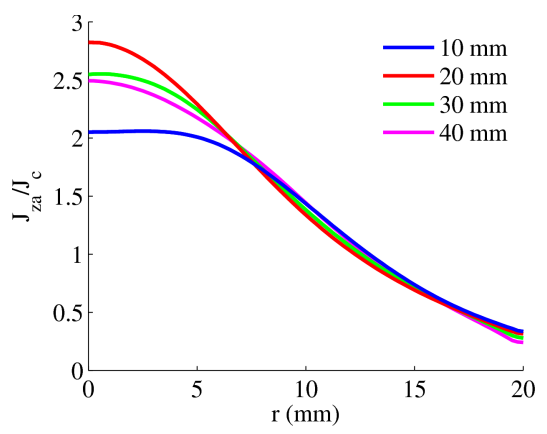

(b)

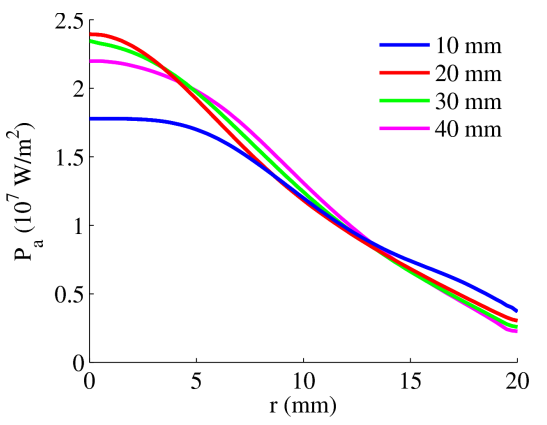

Fig. 5: Radial profile on the anode surface of (a) the axial current density normalized by the cathode current density and (b) the energy flux density transferred to the anode computed for different gap lengths (supersonic arc, $I_{a r c}=$ $1 \mathrm{kA}$ and $\left.B_{A M F}=5 \mathrm{mT}\right)$.

For a gap of $10 \mathrm{~mm}$, the axial current density is roughly uniform (about $2 J_{c}$ ) from the center of the anode to about $r \sim 5 \mathrm{~mm}$. For $r>5 \mathrm{~mm}$, it decreases monotonically to a value close to $0.3 J_{c}$ at the edge of the anode. With 
the increase of the gap length, the profile of the axial current density does not evolve monotonically. When the gap varies from $10 \mathrm{~mm}$ to $20 \mathrm{~mm}$, the axial current density near the axis of symmetry increases significantly (reaching about $2.8 J_{c}$ ), whereas it slightly decreases between $20 \mathrm{~mm}$ and $40 \mathrm{~mm}$. Fig. $5 \mathrm{~b}$ shows the radial profile of the energy flux density transferred to the anode $P_{a}$ for different gap lengths, which has been calculated according to the relations detailed in [10]. The profile of $P_{a}$ strongly depends on the axial current density profile and exhibits thus similar variations as those observed in Fig. 5a. In particular, the energy flux density at the anode centre increases (of about 34\%) when the gap length goes from $10 \mathrm{~mm}$ to $20 \mathrm{~mm}$, whereas it weakly decreases when the gap length is further increased from $20 \mathrm{~mm}$ to $40 \mathrm{~mm}$.

\section{B. Discussion}

In order to understand the evolution of the constriction rate for large gaps shown in Fig. 4, the axial variations at a distance of $R / \sqrt{2}$ from the axis of symmetry of the different terms contributing to the radial current density are represented in Fig. 6, taking as an example the case of $40 \mathrm{~mm}$ gap length.

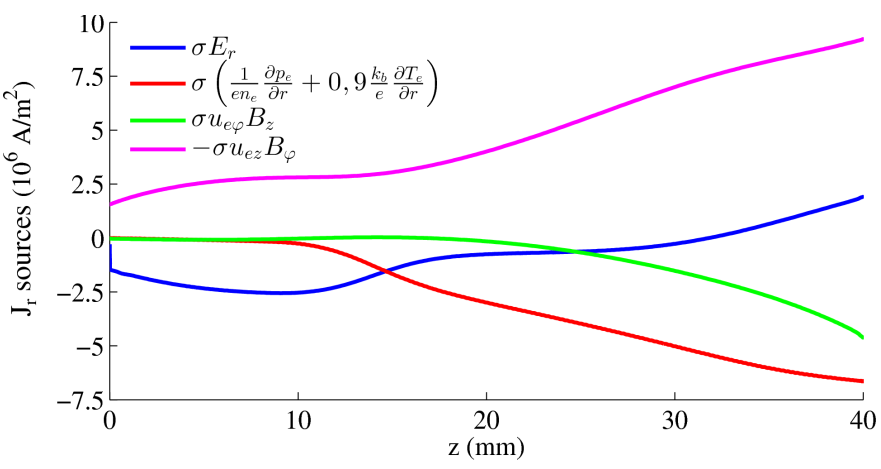

Fig. 6: Axial variations at a distance of $R / \sqrt{2}$ from the axis of symmetry of the different terms contributing to the radial current density for $L_{\text {arc }}=40 \mathrm{~mm}$ (supersonic arc, $I_{\text {arc }}=1 \mathrm{kA}$ and $\left.B_{A M F}=5 \mathrm{mT}\right)$.

Based on the radial projection of the generalized Ohm law (Eq. (3)), the four terms contributing to the radial current density are:

- a term depending on the radial electric field: $\sigma E_{r}$,

- a term resulting from Lorentz forces induced by the azimuthal magnetic field: $-\sigma u_{e z} B_{\varphi}$.

- a term resulting from Lorentz forces related to the AMF: $\sigma u_{e \varphi} B_{z}$

- a term depending on the electron pressure and temperature radial gradients: $\frac{\sigma}{e}\left(\frac{1}{n_{e}} \partial_{r} p_{e}+0.9 k_{b} \partial_{r} T_{e}\right)$. The main component of this term is the first one, related to electron compressibility. The second one has only a corrective effect.

Note that the four terms are proportional to the electrical conductivity. Although the electrical conductivity (which is a function of only the electron temperature) increases in the axial direction, this evolution does not influcence the sign of $\partial \tau_{c} / \partial z$.

One can see from Fig. 6 that the current constriction is almost exclusively caused by the term resulting from Lorentz forces induced by the azimuthal magnetic field (called hereafter the constriction term). The three other terms have, except very close to the anode for the term related to the electric field, an opposing effect restraining the constriction. The three phases of the axial evolution of the constriction rate identified in Fig. 4 may be interpreted as follows. Close to the cathode, only the constriction term and the term related to the electric field play a significant role. The term related to the electric field almost totally offsets the constriction term, resulting in a weak growth of the constriction rate in this region. From $z \sim 11 \mathrm{~mm}$, the reduction of the electric field related term is neutralized by the increase of the term related to electron compressibility. The next phase, characterized by a quick growth of the constriction rate, is due to the important increase of the constriction term, such increase being only partially offset by the rise of the term related to electron compressibility. Finally, close to the anode, the slowing down of the growth of the constriction rate followed by the reduction of the constriction rate is caused by the action of the term related to the AMF, which becomes more and more important when approaching the anode.

In order to interpret some of the results observed in Fig. 6, it is of interest to analyze the distribution in the interelectrode region of the ion density (which is proportional to the electron density given the electroneutrality assumption) and of the streamlines related to the ion flow, which are both shown on Fig. 7 .

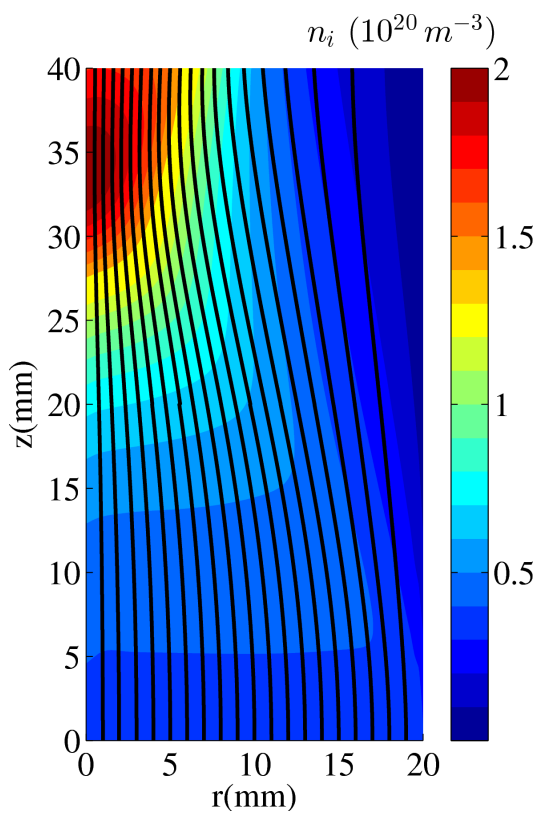

Fig. 7: Distribution of ion density $n_{i}$ and streamlines of the ion flow for $L_{\text {arc }}=40 \mathrm{~mm}$ (supersonic arc, $I_{\text {arc }}=1 \mathrm{kA}$ and $B_{A M F}=5 \mathrm{mT}$ ). The coordinate $\mathrm{z}=0$ corresponds to the cathode surface. 
One can see that the plasma is radially confined in a large region around the axis of symmetry, starting at a distance of around $10 \mathrm{~mm}$ from the cathode and ending at a distance of around $5 \mathrm{~mm}$ from the anode. On the other hand, the plasma undergoes an expansion near the lateral boundary and close to the anode surface near the axis of symmetry. This figure allows to understand the rapid increase of the constriction term starting from a distance of around $15 \mathrm{~mm}$ from the cathode. This effect results from the rapid increase of the electron axial velocity in the constriction term caused by the expansion phenomenon. Indeed, considering the vertical plane located at a radial distance $R / \sqrt{2} \sim 14 \mathrm{~mm}$, the distance of around $15 \mathrm{~mm}$ from the cathode coincides roughly with the transfer of the electrons from the compression region to the expansion region. Since the flow of electrons is mostly axial, the variation of their axial velocity is proportional as a first approximation to $1 / n_{e}$, hence the important increase of the electron axial velocity in the expansion region.

Close to the anode, the term related to the AMF, which limits the constriction, becomes increasingly important. It is of interest to notice that the AMF effect is related to the current constriction process. The electron azimuthal velocity, which comes into play in the term related to the AMF effect $\left(\sigma u_{e \varphi} B_{z}\right)$, is essentially controlled by the azimuthal current density, as this velocity is defined by $u_{e \varphi}=-J_{\varphi} / e n_{e}+u_{i \varphi}$ and the ion azimuthal velocity remains small compared to the electron azimuthal velocity. The azimuthal current density can be expressed from Ohm law by:

$$
J_{\varphi}=\sigma\left(u_{e z} B_{r}-u_{e r} B_{z}\right)
$$

Given the cylindrical symmetry, the radial component of the self-magnetic field is governed only by the azimuthal current density (this results from the azimuthal projection of the Maxwell-Ampere equation). As a consequence, under the presence of an AMF the azimuthal current density (and hence the electron azimuthal velocity) can only start developing under the effect of the second term in Eq. (21). This term is related, through the electron radial velocity and given the fact that the current is mainly carried by electrons, to the evolution of the radial current density (and thus to current constriction). Therefore, the existence of the term related to the AMF is correlated to the current constriction process. Note that very close to the anode, where the constriction rate decreases, the increase of the Lorentz term related to the AMF can not be governed by the second term in Eq. (21). It results from the first term of Eq. (21), whose existence is a consequence of the consideration of the radial component of the selfmagnetic field.

\section{Subsonic arc}

In this section, the behavior of a subsonic diffuse arc between two contacts of $20 \mathrm{~mm}$ radius with a current $I_{\text {arc }}=9 \mathrm{kA}$ and controlled by a uniform AMF of strength $B_{A M F}=45 \mathrm{mT}$ is simulated for a range of gap lengths varying from $10 \mathrm{~mm}$ to $40 \mathrm{~mm}$. The simulations have been performed setting at the boundary between the cathode and the plasma the ion temperature to $3 \mathrm{eV}[2]$, the ion erosion rate to $50 \mu \mathrm{g} . \mathrm{C}^{-1}$ [23] and the electron temperature to $1.5 \mathrm{eV}$ [25].

\section{A. Results}

The norm and the streamlines of the projection of the current density vector onto the $(\mathrm{r}, \mathrm{z})$ plane for $10 \mathrm{~mm}$ and $40 \mathrm{~mm}$ gaps are shown in Fig. 8. For $10 \mathrm{~mm}$, like in Fig.3a, the current starts to constrict in front of the cathode and the constriction becomes more intense in direction to the anode. However, contrary to the case of supersonic conditions, the constriction does not weaken when getting very close to the anode. For $40 \mathrm{~mm}$, in a significant part of the interelectrode region (up to $z \sim 25 \mathrm{~mm}$ ) no current constriction is observed as the current density does not vary much. A constriction of the current is observed only from a distance from the cathode of $25 \mathrm{~mm}$. The constriction then continuously increases up to the anode.

(a)
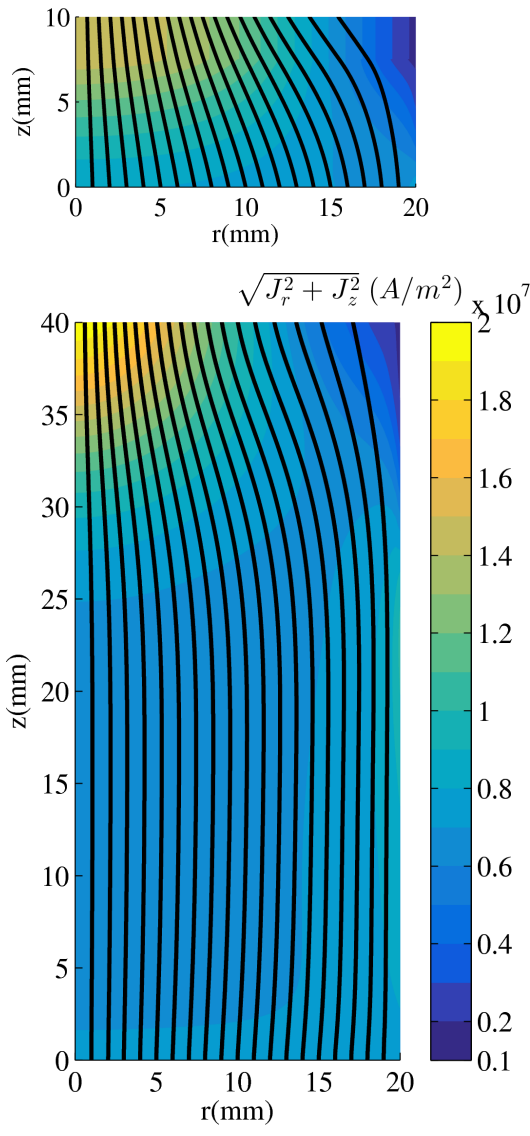

Fig. 8: Distribution of the norm and streamlines of the projection of the current density vector onto the $(\mathrm{r}, \mathrm{z})$ plane for (a) $L_{a r c}=10 \mathrm{~mm}$ and (b) $L_{\text {arc }}=40 \mathrm{~mm}$ (subsonic arc, $I_{\text {arc }}=9 \mathrm{kA}$ and $B_{A M F}=45 \mathrm{mT}$ ). The coordinate $\mathrm{z}=0$ corresponds to the cathode surface.

Fig. 9 shows the axial profile of the constriction rate $\tau_{c}$ computed for different gap lengths. Like in Fig. 4, $\tau_{c}=0.5$ at the cathode boundary because of the uniform 
distribution of the current density on that surface. For a $10 \mathrm{~mm}$ gap, similarly to what was observed in Fig. 4 for supersonic conditions, $\tau_{c}$ increases from the cathode to the anode. Its growth rate tends to get larger in direction to the anode. At the anode boundary the constriction rate reaches 0.73 . For a gap in the $20-40 \mathrm{~mm}$ range, the evolution of the constriction rate differs significantly from that observed under supersonic conditions. It can be divided into two phases. During the flow of the plasma from the cathode to the anode, $\tau_{c}$ first slowly decreases. During this phase, the constriction rate evolution is not depending on the gap length. Next, the constriction rate grows and reaches 0.75 at the anode whatever the gap length value. This second phase starts at a distance of about $15 \mathrm{~mm}$ from the anode irrespective of the gap length value.

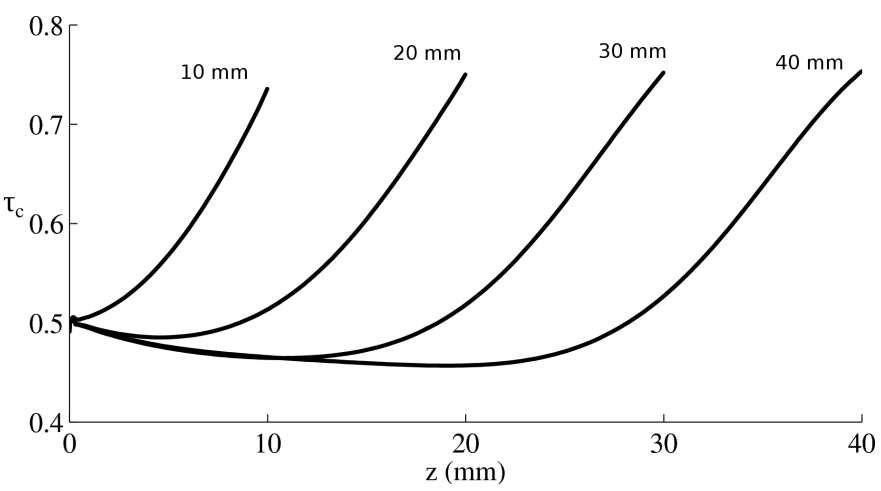

Fig. 9: Axial profile of the constriction rate $\tau_{c}$ for different gap lengths (subsonic arc, $I_{a r c}=9 \mathrm{kA}$ and $B_{A M F}=$ $45 \mathrm{mT})$.

The radial profile of the axial current density on the anode surface for different gap lengths is shown in Fig. 10a. For a contact gap of $10 \mathrm{~mm}$, the axial current density is maximal at the anode center (about $2 J_{c}$ ) and decreases towards the edge of the anode to a value close to $0.3 J_{c}$. The increase of the gap length from $10 \mathrm{~mm}$ to $20 \mathrm{~mm}$ causes a significant increase (of 35\%) of the axial current density at the center of the anode. On the other hand, when the gap length varies from $20 \mathrm{~mm}$ to $40 \mathrm{~mm}$, the profile of the axial current density does not evolve significantly. Similarly to the case of a supersonic arc, the evolution with the gap length of the radial profile of the energy flux density transferred to the anode (Fig. 10b) is close to that of the radial profile of the axial current density collected at the anode surface, since the energy flux density transferred to the anode is strongly correlated to the current density. In particular, the increase of the gap length from $10 \mathrm{~mm}$ to $20 \mathrm{~mm}$ leads to a moderate increase (about 17\%) of the maximum value of the energy flux density, which is reached at the anode centre. On the other hand, the variation of the gap length from $20 \mathrm{~mm}$ to $40 \mathrm{~mm}$ has no effect on the profile of the energy flux density. (a)

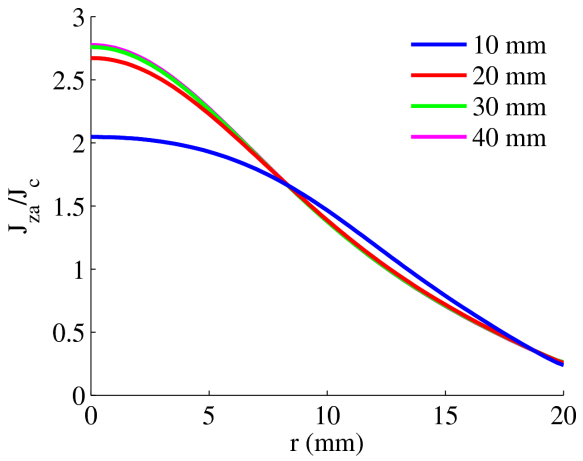

(b)

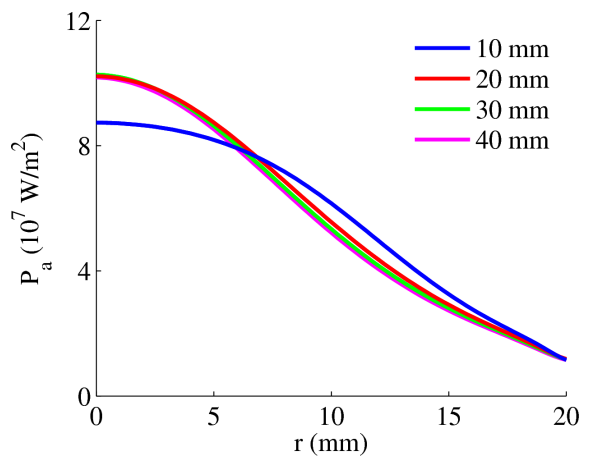

Fig. 10: Radial profile on the anode surface of (a) the axial current density normalized by the cathode current density and (b) the energy flux density transferred to the anode computed for different gap lengths (subsonic arc, $I_{\text {arc }}=9 \mathrm{kA}$ and $B_{A M F}=45 \mathrm{mT}$ ).

\section{B. Discussion}

Following the same approach as for supersonic conditions, Fig. 11 shows for subsonic conditions the axial variations at a distance of $R / \sqrt{2}$ from the axis of symmetry of the different terms contributing to the radial current density, taking as an example the case of $40 \mathrm{~mm}$ gap length.

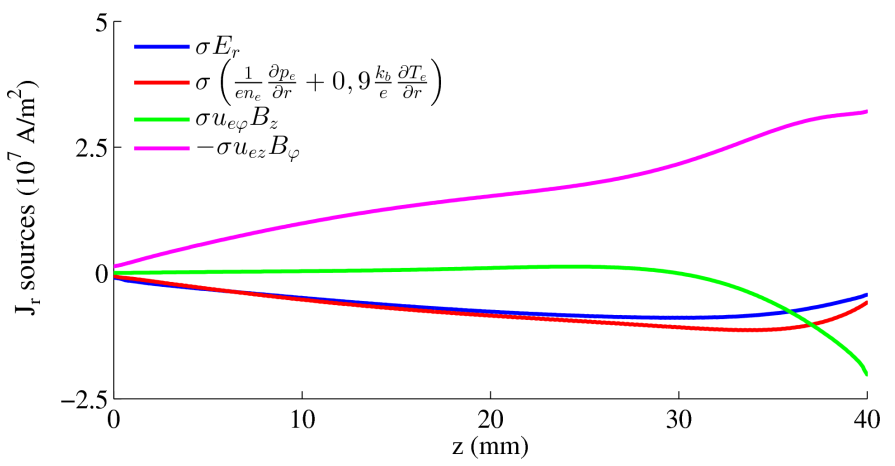

Fig. 11: Axial variations at a distance of $R / \sqrt{2}$ from the axis of symmetry of the different terms contributing to the radial current density for $L_{\text {arc }}=40 \mathrm{~mm}$ (subsonic arc, $I_{\text {arc }}=9 \mathrm{kA}$ and $\left.B_{A M F}=45 \mathrm{mT}\right)$.

Note that, similarly to the supersonic conditions, the current constriction is exclusively caused by the term resulting from Lorentz forces induced by the azimuthal 
magnetic field (called hereafter the constriction term). Based on this figure, the two phases of the axial evolution of the constriction rate identified in Fig. 9 may be interpreted as follows. The first phase, taking place from the cathode surface up to a distance from the anode of around $15 \mathrm{~mm}$, was characterized by a slow decrease of the constriction rate. During this phase, the progresive growth of the constriction term is counterbalanced by the increase of the terms related to the electric field and to electron compressibility. The sum of these two latter terms slightly exceeds the constriction term, resulting in the slow decrease of the constriction rate. Moreover, during this phase the term related to the AMF remains negligible. The second phase, taking place close to the anode (from a distance from the anode of around $15 \mathrm{~mm}$ ), was characterized by a rapid increase of the constriction rate. This is related to the rapid increase of the constriction term, whereas the terms related to the electric field and to electron compressibility become less important. During this phase, the term related to the AMF, which acts against the constriction, comes into play and becomes more and more important. Yet, it is not sufficient to counterbalance the growth of the constriction term.

Our conclusion about the absence of an appreciable evolution of the current constriction at the anode surface for gap lengths greater than $20 \mathrm{~mm}$ is in conflict with Han et al. simulation results, that show a monotonic increase of the constriction with the contact gap [15]. The model employed by Han et al. is similar to ours. Yet various differences between both models can be identified. Han model does not consider the radial and axial components of the self-magnetic field and uses a slightly different formulation of the anode boundary condition for the ion pressure. Moreover, there are also significant differences in the simulation parameters considered in both studies. In Han et al. simulations, the ion temperature at the cathode boundary is much larger (by more than a factor 3 ), the contact radius is $50 \%$ greater and the current density and the strength of the AMF with respect to the arc current are respectively $26 \%$ and $20 \%$ smaller. The precise reason of the different influence of the gap length on current constriction at the anode predicted by both models remains unclear. One may speculate that the current density and/or the AMF strength in [15] may be too small to lead to the saturation of the current constriction at the anode surface.

\section{Conclusion}

Current constriction in a diffuse vacuum arc discharge controlled by an AMF for large gap lengths (up to $40 \mathrm{~mm}$ ) was studied numerically using a two temperature MHD model for both supersonic and subsonic flow conditions.

For both flow conditions, current constriction at the anode surface increases significantly when the gap length grows from $10 \mathrm{~mm}$ to $20 \mathrm{~mm}$. When the gap is further increased, the constriction slightly decreases for a supersonic VA, while it no longer evolves for a subsonic VA. The mechanisms responsible for the current constriction are different according to the flow conditions. In a supersonic VA, the constriction starts developing from the cathode surface and increases in the interelectrode region except close to the anode. The increase of the current constriction is partly correlated to the radial confinement of the plasma caused by Lorentz forces. In a subsonic VA, the constriction starts developing only close to the anode (at a distance of around $15 \mathrm{~mm}$ ) under the influence of the anode sheath. In the rest of the interelectrode region, the current density is subjected to only little variation. For a short gap $(10 \mathrm{~mm})$, the influence of the anode sheath is already effective at the cathode surface and the constriction happens immediately.

The increase of the current constriction at the anode surface when varying the gap length from $10 \mathrm{~mm}$ to $20 \mathrm{~mm}$ causes an increase of the energy flux density from the plasma to the center of the anode, but this increase remains moderate (respectively $34 \%$ and $17 \%$ for supersonic and subsonic VAs). For larger contact gaps, the variation of the gap length has no appreciable influence on the profile of the energy flux density from the plasma to the anode. This suggests that the risk of excessive heating of the anode and of anode spot development is not significantly increased for arcs with very large gaps.

The present study was concerned with vacuum arcs controlled by an homogeneous AMF. However, it has been reported that for large gaps the AMF induced by the contacts may be weaker around the center of the interelectrode region, causing instabilities in the VA behaviour [26]. Arc shape may change fastly and become not axisymmetric. Consequently, future studies on VA at large contact gaps will need to take into account 3D geometry effects and unsteady phenomena.

\section{Acknowledgments}

This project was supported by the French Government in the framework of the "Investments for the Future" programme under the project reference (ANE-ITE-002$01)$.

\section{References}

[1] R. L. Boxman, S. Goldsmith and A. Greenwood, "Twenty-Five Years of Progress in Vacuum Arc Research and Utilization", IEEE Trans. Plasma Sci., vol. 25, no. 6, pp. 1174-1186, 1997.

[2] E. Schade and D. L. Shmelev, "Numerical Simulation of HighCurrent Vacuum Arcs With an External Axial Magnetic Field", IEEE Trans. Plasma Sci., vol. 37, no. 5, pp. 693-697, 2003.

[3] P. Picot, "Vacuum Switching. Technical Report 198", Schneider Electric, 2000

[4] X. Godechot, S. Chakraborty, A. Girodet and P. Vinson, "Design and Tests of Vacuum Interrupters for High Voltage Circuit Breakers", in Proceedings of XXVIth Int. Symp. on Discharges and Electrical Insulation in Vacuum, Bombay India, pp. 417-420, 2014.

[5] E. Schade and D. L. Shmelev, "Numerical Simulation of HighCurrent Vacuum Arcs in External Magnetic Fields", in Proceedings of XXIth Int. Symp. on Discharges and Electrical Insulation in Vacuum, Yalta - Ukraine, pp. 411-414, 2004.

[6] L. Wang, S. Jia, Z. Shi and M. Rong, "Numerical Simulation of Vacuum Arc under Different Axial Magnetic Fields", J. Phys. D: Appl. Phys., vol. 38, no. 7, pp. 1034-1041, 2005. 
[7] Y. I. Londer and K. N. Ulyanov, "Mathematical Model of the Vacuum Arc in an External Axial Magnetic Field", IEEE Trans. Plasma Sci., vol. 35, no. 4, pp. 897-904, 2007.

[8] W. Hartmann, A. Hauser, A. Lawall, R. Renz and N. Wenzel, "Development of a FEM Simulation of Axial Magnetic Field Vacuum Arcs", in Proceedings of XXIIIrd Int. Symp. on Discharges and Electrical Insulation in Vacuum, Bucharest Roumania, pp. 398-401, 2008.

[9] J. Jadidian, "A Compact Design for High Voltage Direct Current Circuit Breaker", IEEE Trans. Plasma Sci., vol. 37, no. 6, pp. 1084-1091, 2009.

[10] Y. Langlois, P. Chapelle, A. Jardy and F. Gentils, "On the Numerical Simulation of the Diffuse Arc in a Vacuum Interrupter", J. Appl. Phys., vol. 109, no. 11, pp. 3306-3318, 2011.

[11] L. Wang, S. Jia, Q. Zhou, W. Haijing and Z. Shi, "ThreeDimensional Model and Simulation of Vacuum Arcs under Axial Magnetic Fields", Phys. Plasmas, vol. 19, no. 1, pp. 3507-3517, 2012.

[12] N. Wenzel, A. Lawall, U. Schümann and S. Wethekam, "Combined Experimental and Theoretical Study of Constriction Threshold of Large-Gap AMF Vacuum Arcs", in Proceedings of the XXVIth Int. Symp. on Discharges and Electrical Insulation in Vacuum, Bombay-India, pp. 193-196, 2014.

[13] L. Wang, Q. Zhou, X. Huang, S. Jia and Z. Shi, "ThreeDimensional Time-Dependent Model and Simulation of HighCurrent Vacuum Arc in Commercial Axial Magnetic Field Vacuum Interrupter", IEEE Trans. Plasma Sci., vol. 41, no. 8, pp. 2015-2021, 2013.

[14] L. Wang, X. Huang, S. Jia, J. Deng, Z. Qian and Z. Shi, "3D Numerical Simulation of High Current Vacuum Arc in Realistic Magnetic Fields Considering Anode Evaporation", J. Appl. Phys., vol. 117, no. 24, pp. 3301-3314, 2015.

[15] W. Han, Z. Yuan and J. He, "Variation of Plasma Parameters of Vacuum Arc Column with Gap Distance", Jpn. J. Appl. Phys., vol. 55, no. 7, pp. 6001-6009, 2016.

[16] Y. Zhang, X. Yao, Z. Liu, Y. Geng, and P. Liu, "Axial Magnetic Field Strength Needed for a $126-\mathrm{kV}$ Single-Break Vacuum Circuit Breaker during Asymmetrical Current Switching", IEEE Trans. Plasma Sci., vol. 41, no. 6, pp. 1670-1678, 2013.

[17] B. Tezenas du Montcel, P. Chapelle, C. Creusot, and A. Jardy, "Arc Appearance and Cathode Spot Distribution in a Long Gap High-Current Vacuum Arc Controlled by an External Axial Magnetic Field", IEEE Trans. Plasma Sci., vol. 46, no. 10, pp. 3722-3730, 2018.

[18] S. I. Braginskii, "Transport Processes in Plasma", Rev. Plasma Phys., vol. 1, pp. 205-311, 1965.

[19] J. Kutzner and H. C. Miller, "Integrated Ion Flux Emitted from the Cathode Spot Region of a Diffuse Vacuum Arc", J. Phys. D: Appl. Phys., vol. 25, no. 4, pp. 686-693, 1992.

[20] D.B. Zolotukhin, S. Hurley and M. Keidar,"Anode Ablation and Performance Improvement of Microcathode Arc Thruster", Plasma Sources Sci. Technol., vol. 28, 034001, 2019.

[21] C. Wieckert and W. Egli, "Theoretical Analysis of the Current and Energy Flow to the Anode in the Diffuse Vacuum Arc, IEEE Trans. Plasma Sci., vol. 17, no. 5, pp. 649-652, 1989.

[22] K. U. Riemann, "The Bohm Criterion and Sheath Formation", J. Phys. D: Appl. Phys., vol. 24, no. 4, pp. 493-518, 1991.

[23] J. E. Daalder, "Components of Cathode Erosion in Vacuum Arcs", J. Phys. D: Appl. Phys., vol. 9, no. 16, pp. 2379-2395, 1976.

[24] I. I. Beilis and M. P. Zektser, "Calculation of the Parameters of the Cathode Stream of an Arc Discharge", High Temp., vol. 29, pp. 501-504, 1991.

[25] G. A. Lyubimov and V. I. Rakhovskii, "The Cathode Spot of a Vacuum Arc", Sov. Phys. Uspekhi, vol. 21, no. 8, pp. 693-718, 1978.

[26] S. Cheng and J. Wang, "Study on High-Current Vacuum Arc Characteristics Under Self-Generated Axial Magnetic Field of Contact at a Long Contact Gap for High-Voltage Vacuum Interrupters", IEEE Trans. Plasma Sci., vol. 37, no. 1, pp. 243$253,2009$. 